\title{
Disseminated and sustained HIV-infection in CD34+ cord blood cell transplanted Rag2-/-gc-/- mice \\ Stefan Baenziger ${ }^{1}$, Roxane Tussiwand ${ }^{2}$, Erika Schlaepfer ${ }^{1}$, Luca Mazzucchelli ${ }^{3}$, Mathias Heikenwalder ${ }^{1}$, Michael O Kurrer ${ }^{1}$, Silvia Behnke ${ }^{1}$, Joachim Frey ${ }^{4}$, Annette Oxenius ${ }^{5}$, Helen Joller ${ }^{1}$, Adriano Aguzzi ${ }^{1}$, Markus G Manz ${ }^{2}$ and Roberto F Speck*1
}

\author{
Address: ${ }^{1}$ University Hospital of Zurich, Zurich, Switzerland, ${ }^{2}$ Institute for Research in Biomedicine, Bellinzona, Switzerland, ${ }^{3}$ Institute for \\ Pathology, Locarno, Switzerland, ${ }^{4}$ University of Berne, Berne, Switzerland and ${ }^{5}$ Swiss Federal Institute of Technology, Zurich, Switzerland \\ * Corresponding author
}

from 2006 International Meeting of The Institute of Human Virology Baltimore, USA. 17-21 November, 2006

Published: 21 December 2006

Retrovirology 2006, 3(SuppI I):S3I doi:I0.1 186/I742-4690-3-SI-S3I

(c) 2006 Baenziger et al; licensee BioMed Central Ltd.

\section{Background}

We evaluated a novel mouse model that is based on the transplantation of human cord blood CD34+cells into immunodeficient Rag2-/-gc-/- newborn mice for studying HIV.

\section{Results}

Reconstituted mice (CD45+ cell engraftment: $29 \pm 18 \%$ ) were permissive to HIV with up to $2 \times 10^{6}$ copies $/ \mathrm{ml} \mathrm{2-6}$ weeks after infection. Thereafter, viremia stabilized at lower levels for up to 4 months. A marked CD4+ cell depletion occurred in all mice infected with X4- strains simultaneously with the initial rise of plasma HIV RNA while this was not the case in R5-infected mice. Spleens and lymph nodes of mice infected with either R5- or X4strains contained p24+ cells. In thymi, however, p24+ cells were detected rather exclusively following infection with X4- strain, consistent with the expression of CXCR4 but not CCR5 on human CD4+ thymocytes. Similarly as in humans, HIV-infected macrophages were only occasionally found.

\section{Conclusion}

Rag2-/-gc-/- mice transplanted with human CD34+ cells develop long-term, high-titer, and lymphoid organ disseminated infection irrespective of co-receptor selectivity of HIV strain, closely resembling HIV infection in man. In particular, by using HIV strains with distinct co-receptor selectivity, we clearly illustrate the higher cytopathic potential of X4- strains as compared to R5- strains. This straightforward to generate and cost-effective in vivo model should be valuable to study virus-induced pathology, and to rapidly evaluate new approaches aiming to prevent or treat HIV infection. 